\title{
Black hole superradiance as a probe of ultra-light new particles
}

\author{
Robert Lasenby \\ Perimeter Institute for Theoretical Physics, 31 Caroline Street N, Waterloo, Ontario N2L 2Y5, \\ Canada \\ email: rlasenby@perimeterinstitute.ca
}

\begin{abstract}
Bosonic fields around a spinning black hole can be amplified via 'superradiance', a wave analogue of the Penrose process, which extracts energy and momentum from the black hole. For hypothetical ultra-light bosons, with Compton wavelengths on $\gtrsim \mathrm{km}$ scales, such a process can lead to the exponential growth of gravitationally bound states around astrophysical Kerr black holes. If such particles exist, as predicted in many theories of beyond Standard Model physics, then these bosonic clouds give rise to a number of potentially-observable signals. Among the most promising are monochromatic gravitational radiation signals which could be detected at Advanced LIGO and future gravitational wave observatories.
\end{abstract}

\section{Overview}

Astrophysical and cosmological evidence, along with the theoretical problems of Planckscale physics, point towards new physics beyond the Standard Model (SM). However, direct evidence, such as signals in laboratory experiments, has so far been elusive. This may be because any new particles have masses too heavy to be produced by existing experiments such as the LHC. Hypothesised examples include the supersymmetric partners of SM particles (though in many models these should have been observed at the LHC), or the extra particles predicted by Grand Unified Theories. Alternatively, it is possible that new light states exist, but couple very weakly to SM matter, so that their effects are weak enough to have been undetected, similarly to how the neutrino was not detected until 60 years ago. Such new particles arise naturally in many models of Beyond Standard Model physics — examples include the QCD axion, string theory axions, 'fifth forces', and many others (see e.g. Essig et al. 2013).

The one way in which any new particles must interact with SM states is through gravity. However, the spacetime curvature scales achieved in the experimentally-accessible universe are $\gtrsim \mathrm{km}$, which corresponds to a very low effective source density in particle physics terms. For example, a 10 Solar mass black hole has a Hawking temperature of $T_{H}=1 /\left(8 \pi G M_{\mathrm{BH}}\right) \sim 10^{-8} \mathrm{~K}\left(10 M_{\odot} / M_{\mathrm{BH}}\right)$ (using natural units). Gravitational production in particle physics process is suppressed by multiple powers of the energy of the process over the Planck mass, again giving tiny effects.

One way to get around the very small spacetime curvatures available is to take advantage of coherence enhancement - as in a laser, this can give rise to an exponential amplification of a very small initial population. The 'black hole bomb' thought experiment of Press \& Teukolsky (1972) used the wave analogue of the Penrose process, 'superradiant scattering', through which energy and angular momentum can be extracted from a spinning black hole. Along with a mirror to obtain multiple passes of amplification, this gives an exponentially growing wave around the hole. The key idea behind using black hole superradiance to probe new light particles is that, if they have a non-zero mass, such particles will form bound states around the black hole, acting as 'their own 


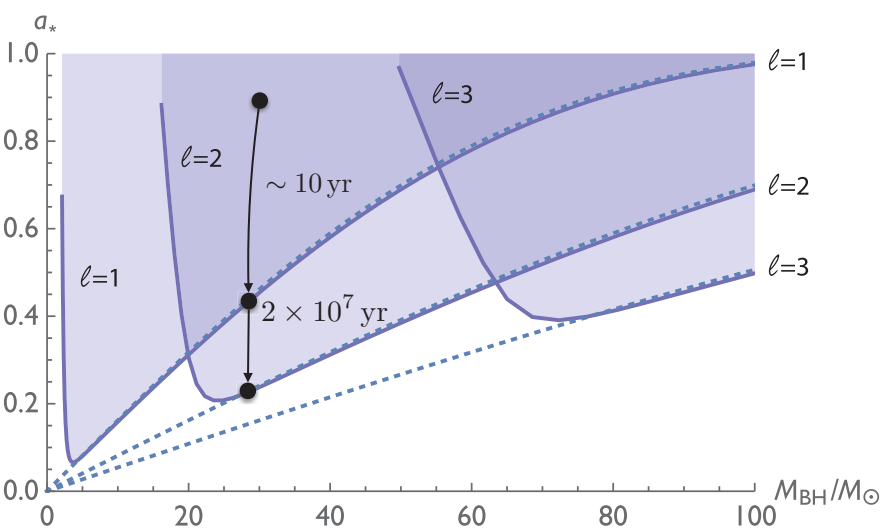

Figure 1. Illustration of how a black hole of initial mass $30 M_{\odot}$ and initial dimensionless spin 0.9 evolves, through superradiance of a spin-0 field with mass $\mu=6 \times 10^{-13} \mathrm{eV}$. The first arrow shows how the angular momentum $l=1$ level grows over a timescale of $\sim 10 \mathrm{yr}$, extracting a significant fraction of the black hole's spin, up to the critical spin at which this bound state is no longer superradiant. The $l=2$ level takes much longer to grow, $\sim 2 \times 10^{7} \mathrm{yr}$, but will still extract all of the available angular momentum on shorter-than-cosmological timescales. The $l \geqslant 3$ levels take longer than the age of the universe to grow, so the black hole will remain on the critical $l=2$ line. Repeating the same story for other initial masses and spins, if a black hole begins life within the shaded region, then it will end up at the boundary of the shaded region over the lifetime of the universe. Since, for the case shown, the boson's Compton wavelength is significantly larger than the size of the black hole, the bound state wavefunctions are mostly localised far away from the hole. Thus, they can extract a significant proportion of the black hole's angular momentum while only extracting a small fraction of its mass, as illustrated by the almost vertical evolution arrows.

mirror' (Detweiler 1980, Arvanitaki et al. 2010). For a spinning black hole, some of these bounds states can be superradiant — rather than decaying into the black hole, they will be amplified with time. Though such growth rates will be much less than the black hole crossing time, and thus extremely small on particles physics scales, if the new particle is bosonic then its occupation number can grow exponentially. Thus, a significant proportion of the black hole's energy and angular momentum can be extracted on a timescale that is short compared to astrophysically-relevant periods.

If such an exponential growth occurred, it could have a number of astrophysical signatures. The most obvious one is that initially fast-spinning black holes would be spun down over time by the formation of a bosonic cloud, as illustrated in Figure 1 (Section 2 goes over this process in more detail). The most accurate spin determinations come from black holes in X-ray binaries. Since, as per Figure 2, a few of these are observed to have near-extremal spins incompatible with significant spin-down, we can rule out the presence of a new light, weakly-coupled boson across a certain range in masses and couplings.

Recently, the LIGO gravitational wave observatory detected its first binary black hole merger, and through analysis of the waveform, was able to put some constraints on the masses and spins of the pre-merger black holes. In the near future, upgraded gravitational wave observatories will likely detect hundreds more such mergers. Though the errors on each individual spin measurement will be fairly large, the statistical information from many such events may provide indications of a spin-down effects, if one exists (since we do not know the pre-merger history of such systems, robust detections or exclusions would be difficult).

Gravitational wave observatories provide another, more promising probe of superradiance - the gravitational waves from the bosonic cloud itself. As explained in Section 2, 


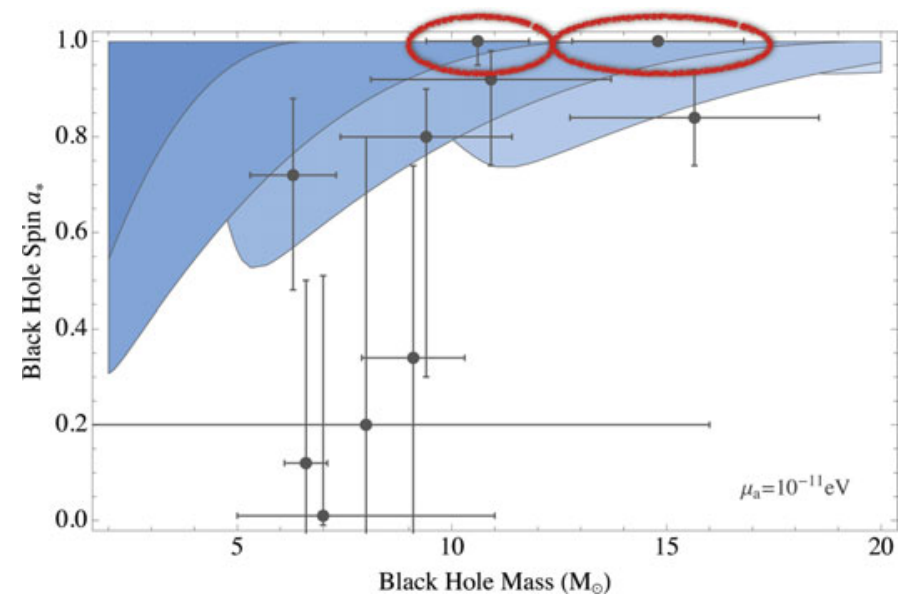

Figure 2. Illustration of how black hole spin measurements can place bounds on the existence of a light spin-0 particle (from Arvanitaki et al. 2015). The shaded areas, as per Figure 1, show the region within which an initially-fast-spinning black hole gets spun down to the boundary of the shaded region (in this case, for a particle of mass $\mu_{a}=10^{-11} \mathrm{eV}$ ). The dots with error bars show the masses and spins of black holes, as measured via X-ray observations of accretion disks (Arvanitaki et al. 2015). The two circled measurements are well within the shaded region, so under the assumption that nothing would have disturbed the growth of a bosonic cloud (which is supported by the measured properties of these systems (Arvanitaki et al. 2015)), a weaklycoupled scalar boson of this mass is ruled out. The spin measurements errors for fast-spinning black holes are very small, since the properties of the accretion disk depend non-linearly on $a_{*}$ at near-extremal spins.

the superradiant growth rate is largest when the boson's Compton wavelength is around the size of the black hole. This means that oscillations of the bosonic field, having a frequency of roughly the boson's mass, are naturally in the LIGO frequency range for roughly-stellar-mass black holes. These oscillations source monochromatic gravitational radiation, at a frequency set (to within a few percent (Arvanitaki et al. 2016)) by the boson's mass.

If there is a black hole with a large bosonic cloud sufficiently close to Earth — which, for most of the parameter space, means being inside our galaxy — then it could be detected as a monchromatic signal at LIGO, similar to that expected from a slightly asymmetric neutron star. However, signals from superradiant clouds would have the added feature that separate signals would be expected to cluster at frequencies within a a few percent of each other (as well as displaying subtly different frequency drift properties from a neutron star). As shown in Figure 3, depending on the mass and spin distribution of black holes, it is possible that such a signal could be seen within the next few years, if there is a light boson in the correct mass range.

The effects discussed so far have assumed that the new boson interacts sufficiently weakly with SM matter, and with itself, that gravity dominates its evolution around the black hole. This is expected to be true for some beyond Standard Model particle candidates - for example, a very light QCD axion also has an extremally small quartic self-coupling — but not for others. When self-interactions become important, very complicated behaviour can occur, though for large enough self-interactions, we would expect superradiant growth to saturate well before extracting a significant fraction of the black hole's energy and angular momentum. 


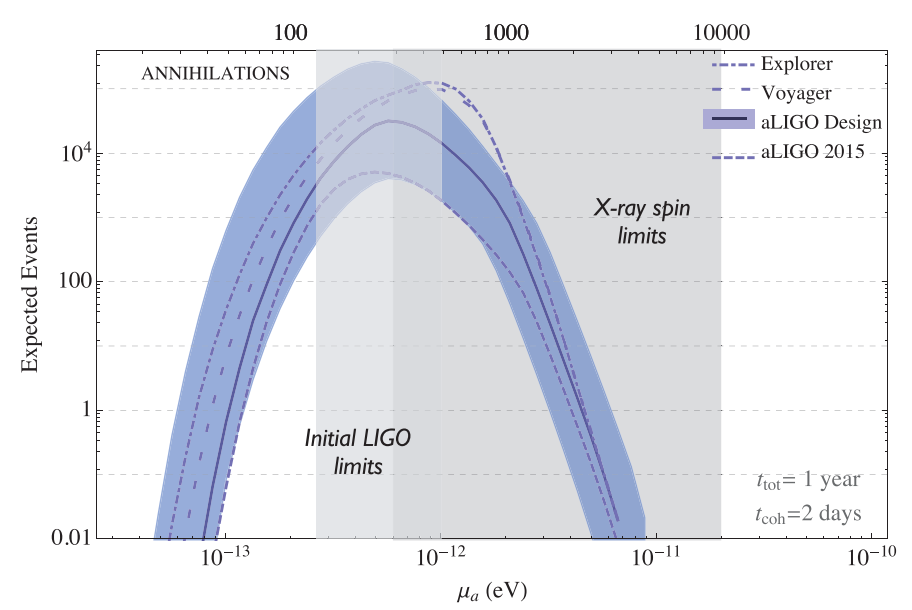

Figure 3. Expected number of monochromatic gravitational wave signals from black hole superradiance of a light scalar, detectable with one year of gravitational wave observations, for realistic black hole mass and spin distributions (from Arvanitaki et al. 2016). The shaded bands correspond to the range between pessimistic and optimistic black hole distribution with Advanced LIGO at design sensitivity. The various dotted lines correspond to gravitational wave detectors with different sensitivies. Boson masses $\mu_{a}$ in the greyed-out region are disfavoured by black hole spin measurements (Figure 2). In the light-gray region, null results from initial LIGO searches mean there is less chance of observing a new signal.

\section{Black hole spin-down by superradiance}

In this section, we give a slightly more detailed account of how the superradiant growth of bosonic bound states around a Kerr black hole can spin it down.

Rotational superradiance - amplification of scattered waves by rotating objects - is not a phenomenon that is unique to black holes. For example, as shown by Zeldovich (1971), a rotating conductor (with some resistivity) can amplify incident electromagnetic waves. The effect is a direct kinematic counterpart to the ability of a non-rotating object to absorb radiation. In the case of a black hole, we can see directly from the area theorem that, in absorbing a particle with $E / L>\Omega_{H}$, where $E$ is the particle's energy, $L$ its angular momentum, and $\Omega_{H}$ the angular velocity of the horizon, the black hole's horizon area would decrease. This is classically disallowed, but the converse is that emitting such a particle leads to an increase in horizon area, so is entropically favoured.

In many situations, we can also estimate the amplification factor (for bound states, the growth rate) from superradiance. Parametrically, the energy flux across the horizon due to a scalar field configuration $\varphi=\varphi_{0} e^{i(\omega t-m \phi)}$ near the horizon is

$$
P \sim A_{H}\left|\varphi_{0}\right|^{2} \omega\left(\omega-m \Omega_{H}\right)
$$

We see the occurrence of the $\omega-m \Omega_{H}$ factor expected from the area theorem - when $\omega / m<\Omega_{H}$, i.e. when the angular phase velocity of the field is less than the angular velocity of the horizon, the energy flux into the hole is negative. Bound states necessarily have $\omega<\mu$, where $\mu$ is the mass of the scalar field, so the bound states with the highest growth rate will generally be those with the largest field values near the horizon, among those with $m>\omega / \Omega_{H}$.

The states most localised near the black hole are those with the lowest energy and angular momentum. If $\mu<\Omega_{H}$ (for a fast-spinning black hole, if the Compton wavelength is large compared to the size of the hole), then all states with $m \geqslant 1$ are superradiant. If $\mu \gg \Omega_{H}$, then only states with $m>\mu / \Omega_{H}$ are superradiant; due to the large angular 


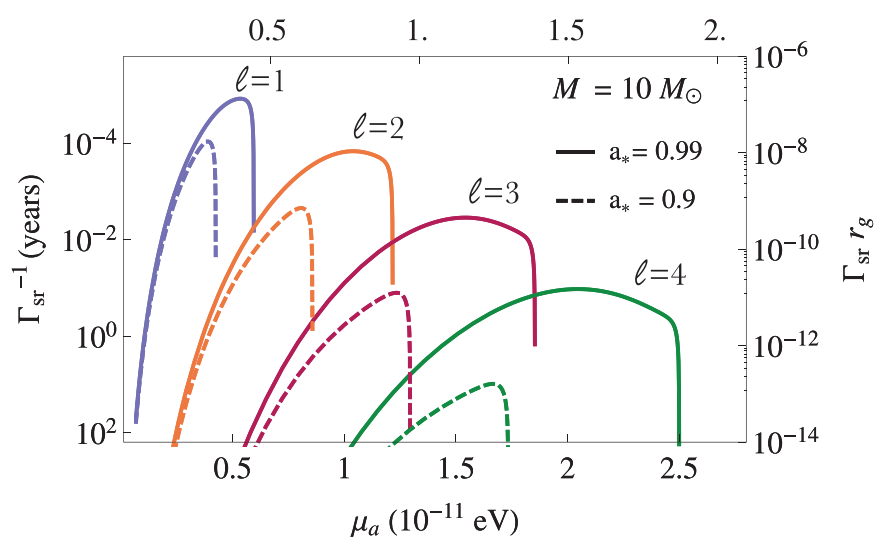

Figure 4. Superradiant growth rates $\Gamma_{\mathrm{sr}}$ for a scalar field around a Kerr black hole of 10 Solar masses, with dimensionless spin 0.99 (solid lines, 0.9 dotted lines), from Arvanitaki et al. 2015. The different curves show the growth rate of the fastest-growing level for each angular momentum value $l$. As discussed in the text, higher-l levels have smaller growth rates, and for each $l$ level, the fastest growth rate occurs for $\mu$ near the maximum allowed for superradiance (the upper horizontal scale shows $G M_{\mathrm{BH}} \mu=\mu r_{S} / 2$ ).

momentum barrier, their value near the horizon is very small. Consequently, as shown in Figure 4, the maximum growth rate is achieved for $\mu \sim 0.4 /\left(G M_{\mathrm{BH}}\right)$ - for lower values, the bound states are localised futher away from the black hole, while for larger values, only higher angular momentum states are superradiant, and their value near the horizon is suppressed by the angular momentum barrier.

Following the above discussion, Figure 1 illustrates how the existence of a light weaklycoupled boson would affect the mass and spin evolution of an initially-fast-spinning black hole. The dotted lines show the critical spin values below which superradiance does not occur, for each angular momentum value $m$. If a black hole begins life above one of these, and the superradiance rate of the fastest-growing level is fast enough, then it will be spun down by the formation of a bosonic cloud, until it is almost on the critical line. This process will then repeat with the next $m$ level, again if the growth rates are fast enough, with the result that we expect all sufficiently old (and undisturbed) black holes to have spins below a mass-dependent value, with initially-fast-spinning holes ending up at these critical spins. As discussed above, such a pattern can be tested or ruled out by black hole spin measurements, in addition to gravitational wave signatures from the bosonic clouds themselves.

\section{Summary}

Black hole superradiance provides a unique probe of light bosons beyond the Standard Model of particle physics, with the ability to exclude or discover arbitrarily weaklycoupled particles in suitable mass ranges. The advent of gravitational wave astronomy opens multiple windows through which such an effect may be discovered, with the potential for observable signals in near-future experiments (Arvanitaki et al. 2015, Arvanitaki et al. 2016).

Acknowledgements: Most of the material in the text is derived from Arvanitaki et al. $20 \overline{15}$ and Arvanitaki et al. 2016. We have not attempted to provide comprehensive references to the extensive literature on black hole superradiance, links to which can be found 
in some of the cited papers. Research at Perimeter Institute is supported by the Government of Canada through Industry Canada and by the Province of Ontario through the Ministry of Economic Development \& Innovation.

\section{References}

Essig et al. 2013, Working Group Report: New Light Weakly Coupled Particles, arXiv:1311.0029 Detweiler 1980, Phys. Rev. D22, 2323

Arvanitaki et al. 2010, Phys. Rev. D81, 123530

Arvanitaki et al. 2011, Phys. Rev. D83, 044026

Arvanitaki et al. 2015, Phys. Rev. D91, 084011

Arvanitaki et al. 2016, arXiv:1604.03958

Press \& Teukolsky 1972, Nature, 238, 211-212

Y. B. Zeldovich 1971, JETP Lett., 14, 180 